\title{
Hématome post-traumatique isolé de la surrénale
}

\section{Post-Traumatic isolated Adrenal Haematoma}

\section{H. Slimani · J. Bidoire $\cdot$ L. Sengler}

Reçu le 5 octobre 2016; accepté le 14 décembre 2016

(C) SFMU et Lavoisier SAS 2016

Une femme de 74 ans, hypertendue, est admise aux urgences suite à une chute mécanique de sa hauteur, responsable d'un traumatisme abdominal fermé. La patiente présentait une douleur abdominale avec vomissements bilieux. L'examen clinique initial retrouvait une défense de l'hypochondre droit. La bandelette urinaire objectivait une hématurie microscopique. Le reste de l'examen clinque était normal. La biologie a montré une hyperleucocytose isolée sans signes de déglobulisation associée à une élévation modérée des transaminases $(1,5 \mathrm{Nl})$. L'échographie abdominale a objectivé une collection liquidienne minime $(42 \mathrm{~mm} \times 21 \mathrm{~mm})$ susrénale et sous-hépatique (Fig. 1A). Le scanner a confirmé la présence d'un hématome de la surrénale droite sans saignement actif (Fig. 1B). Les hématomes post-traumatiques de la surrénale concernent entre $0,03 \%$ et $3 \%$ des cas de traumatisme abdominal [1]. Ils ne constituent pas un facteur de gravité en soi mais en sont un " marqueur » potentiel de par l'association des lésions adjacentes, nécessitant ainsi un bilan lésionnel exhaustif. L'absence de spécificité clinique et biologique démontre tout l'intérêt (diagnostique et pronostique) de l'imagerie, notamment la tomodensitométrie (TDM) lors des traumatismes abdominaux fermés. En effet, la TDM va d'une part évaluer la taille, le volume et l'extension de l'hématome, et d'autre part rechercher d'autres lésions associées. Le traitement conservateur doit être privilégié, bénéficiant ainsi d'une simple surveillance dans la très grande majorité des cas. Il n'y a que lorsqu'il s'associe à un saignement actif qu'il peut bénéficier d'une embolisation. La chirurgie restant exceptionnelle [2]. L'évolution clinique de notre patiente a été satisfaisante avec du repos strict au lit et un traitement antalgique. Le contrôle scannographique réalisé à J4 montrait l'intégrité du rein droit et la stabilité de l'hématome de la surrénale droite.

H. Slimani $(\varangle) \cdot J$. Bidoire $\cdot$ L. Sengler

Service d'accueil des urgences, hôpital Nord-Franche-Comté,

14 rue de Mulhouse, F-90016 Belfort, France

e-mail : hakim.slimani@hnfc.fr

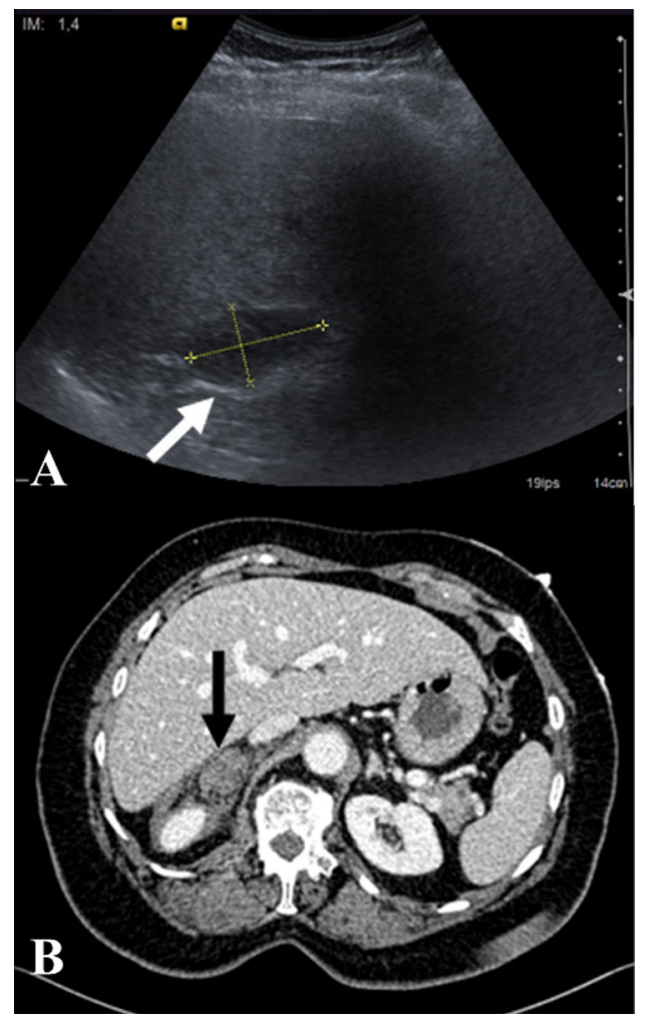

Fig. 1 A : échographie abdominale : collection liquidienne du pôle supérieur du rein droit ; B : TDM abdominal avec injection : hématome de la surrénale droite mesurant $4 \times 3 \times 5 \mathrm{~cm}$, sans saignement actif et petit épanchement hématique dans l'espace de Morrison

\section{Références}

1. Rana AI, Kenney PJ, Lockhart ME, et al (2004) Adrenal gland hematomas in trauma patients. Radiology 230:669-75

2. Chen KT, Lin TY, Foo NP, et al (2007) Traumatic adrenal haematoma: A condition rarely recognised in the emergency department. Injury 38:584-7 